\title{
Simulating the growth of supraglacial lakes at the western margin of the
}

\section{Greenland ice sheet}

\section{Supplementary material: Sensitivity analyses}

Here, we present a selection of sensitivity analyses performed in order to investigate the extent to which the performance of the model is dependent upon the characteristics of the forcing data and key variables used in the flux equations.

\section{S1. Sensitivity to Manning's ' $n$ '}

We use an experimentally derived value for Manning's ' $n$ ' which, for ice, is found to be in the range 0.010-0.012 (Lotter, 1932). We test the sensitivity of the model to Manning's ' $n$ ' within this range and $+/$ - a single order of magnitude: 0.11 and 0.0011 .

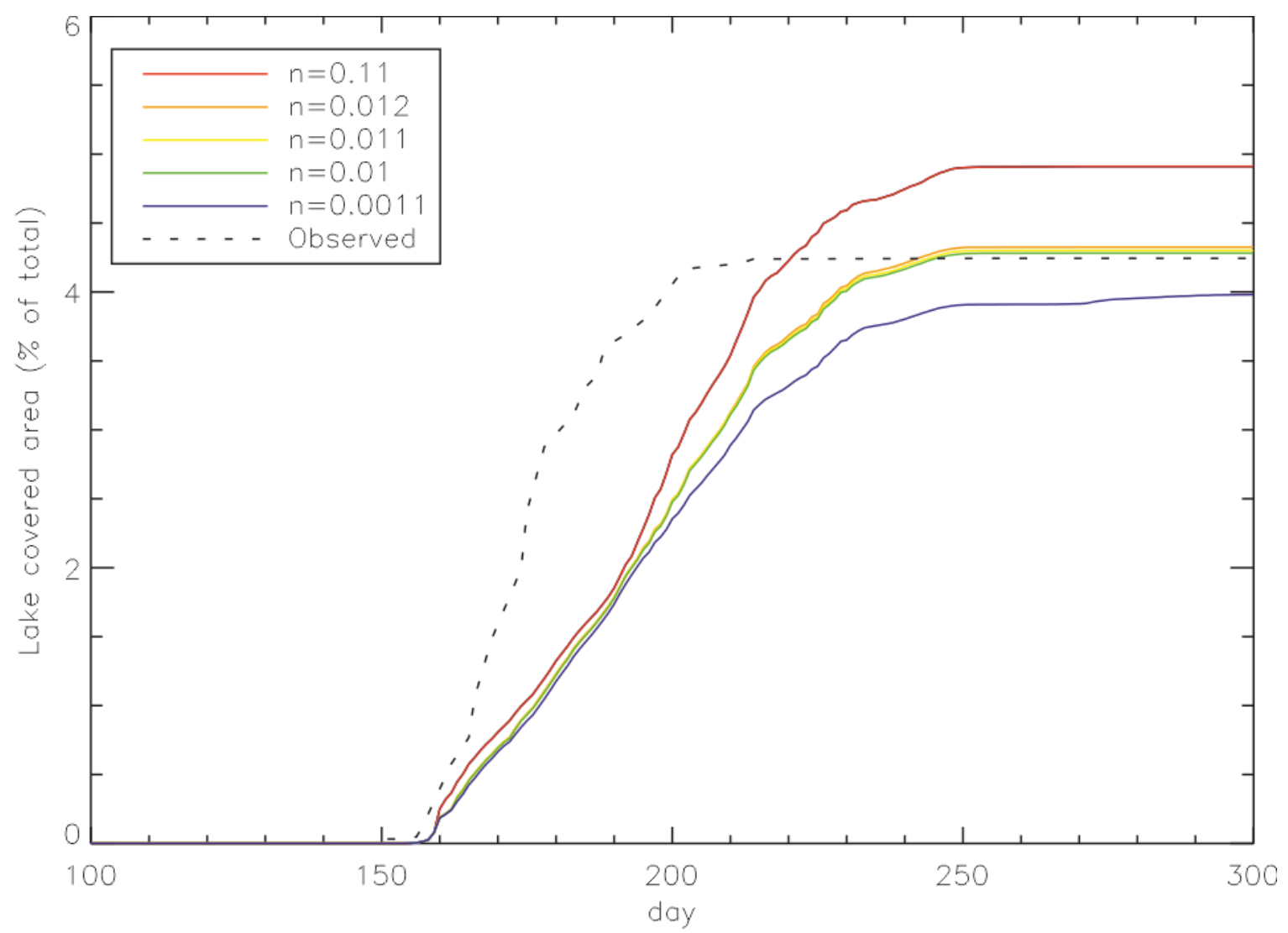

Figure S1: Simulated lake area profiles for a range of ' $n$ ' values (solid). Also shown is the cumulative lake area profile for the observed lakes (dashed). 
Within the range of values proposed by Lotter (1932), we see little variation in simulated supraglacial lake behaviour. If we increase or decrease Manning's ' $n$ ' by an order of magnitude, we see a significant departure from the results obtained using the experimental range, particularly with regards to maximum lake area; when manning's ' $n$ ' is higher lakes grow larger. A Manning's 'n' value of 0.11 is associated with very rough surface conditions; for example river flow over very dense brush or through trees, conversely an ' $n$ ' value of 0.0011 applies to a surface smoother even than polished brass (Chow, 1988). It is clear that flow over bare ice exhibits neither of these roughness characteristics and we feel justified in our choice of 0.011 as the value of Manning's ' $n$ ' in this study.

Table S1: Impact of Manning's 'n' value on model skill.

Manning's ' $\mathrm{n}$ ' comparison

\begin{tabular}{|c|c|c|c|c|c|}
\hline 'n' value & 0.0011 & 0.01 & 0.011 & 0.012 & 0.11 \\
\hline Percentage of observed lakes modelled & 65.69 & 65.69 & 65.69 & 65.69 & 65.69 \\
\hline Odds ratio & 18.18 & 18.12 & 18.23 & 18.22 & 18.56 \\
\hline PSS & 0.31 & 0.32 & 0.33 & 0.33 & 0.35 \\
\hline PSS Standard Error & 0.0028 & 0.0028 & 0.0028 & 0.0028 & 0.0029 \\
\hline Maximum Modelled Lake Area (\%) & 3.98 & 4.28 & 4.30 & 4.33 & 4.91 \\
\hline Maximum Observed Lake Area (\%) & 4.24 & 4.24 & 4.24 & 4.24 & 4.24 \\
\hline Onset date correlation & 75.88 & 75.88 & 75.88 & 75.88 & 75.74 \\
\hline
\end{tabular}

\section{S2. Sensitivity to DEM resolution}

We perform a comparison study to examine the dependence of modelled lake location and area upon the resolution of the digital elevation model (DEM) used as the basis for routing runoff. In order to ascertain the potential of high resolution ice sheet models to resolve supra-glacial lakes, we include a resolution of $1 \mathrm{~km}$ in our study. We reduce the resolution of our DEM (posted at $100 \mathrm{~m}$ ) to $300 \mathrm{~m}, 500 \mathrm{~m}$ and $1000 \mathrm{~m}$ using a neighbourhood averaging method. 

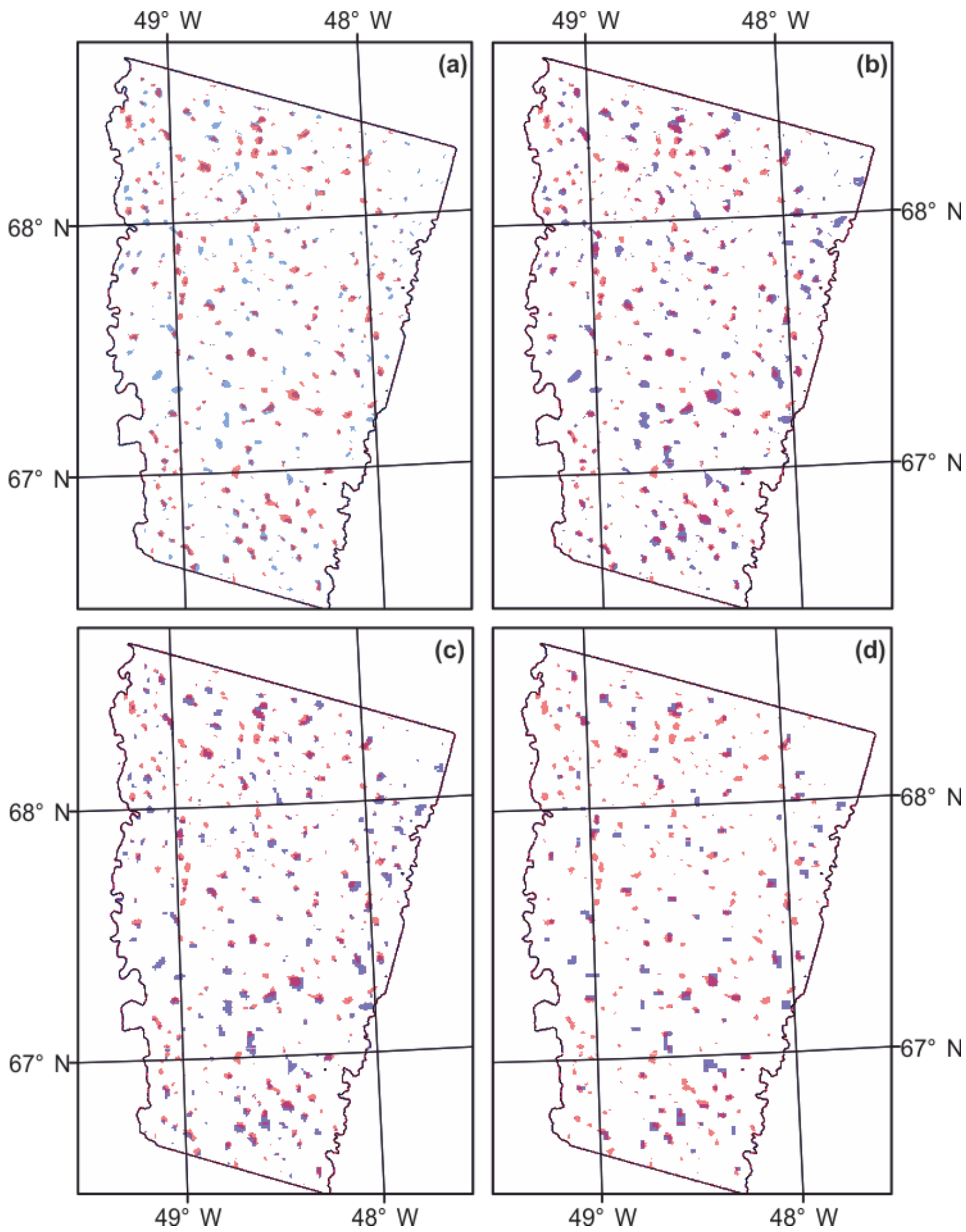

Figure S2: Distribution of simulated lakes (blue) for the year 2003 modelled at a resolution of a: 100 m, b: $300 \mathrm{~m}, c: 500 \mathrm{~m}, \mathrm{~d}: 1000 \mathrm{~m}$. Also shown are MODIS observed lakes (red)and the area coincident in both modelled and observed laked distribution (purple).

The DEM posted at $300 \mathrm{~m}$ performs reasonably well when compared to observations (Figure A2, Table A2). Simulated maximum lake covered area is greater than that using the DEM posted at $100 \mathrm{~m}$. This suggests that the neighbourhood averaging method produces 
depressions in this DEM that are artificially large, with respect to the original DEM. In addition, it is clear that some of the smaller depressions have been smoothed out of the DEM during this process since $48(14 \%)$ less of the observed lakes are reproduced. It is interesting to note that although the $1000 \mathrm{~m}$ resolution DEM reproduces much fewer lakes than the finer resolution models, it does successfully simulate 43 (13\%) of them. The correlation between simulated and observed lake onset day is high using a DEM at all four resolutions. However it is important to remember that this figure is based only on those lakes that the DEM successfully locates, the number of which reduces as the DEM resolution becomes more coarse. We can conclude that although $1000 \mathrm{~m}$ resolution is not sufficient to capture the full range of supra-glacial lake behaviour in terms of water storage (area/volume) or impact on albedo (lake covered area), it could nonetheless be useful, particularly as a guide to potential lake onset day.

Table S2: Impact of the DEM on model skill. These results consider all lakes, even those larger than $0.125 \mathrm{~km}^{2}$.

\begin{tabular}{r|cccc} 
& \multicolumn{5}{|c}{ DEM Posting (m) } \\
\hline Maximum lake covered area (\% total) & 4.5 & 4.9 & 2.8 & 0.9 \\
Number of observed lakes also modelled & 218 & 170 & 116 & 43 \\
Proportion of observed lakes also modelled (\%) & 64 & 50 & 34 & 13 \\
Correlation between simulated and observed & 0.83 & 0.79 & 0.79 & 0.87 \\
onset dates. & & & & \\
\hline
\end{tabular}

\section{S3. Sensitivity to model time-step}

Since free surface gradients are found to be large between some cells, and the maximum travel distance of the water is limited to one cell at each time-step; a fine time-step is needed to represent the full flow path. However, computational expediency prevents the operation of the model at very fine, e.g. 1 second, resolution. The use of a Runge-Kutta approximation to integrate our flow rate equation, over an implicit solution, may contribute to this discrepancy. However, we feel that the principle contribution is from the truncation of flow path when 
using a coarse time-step. In order to test this, we ran the model for one day, day 196 (in the middle of the melt season), using a time-step of 60, 90, 120, 180, 210 and 600 and noted the proportion of cells where displacement greater than water content and production was presented (Figure A3). This incomplete flow occurs in a greater proportion of cells when the time-step is increased. The use of a 600 second time-step meant incomplete flow was observed in over $20 \%$ of cells, which is unacceptable. Using $<0.5 \%$ incomplete flow as an acceptable level of accuracy we choose 90 seconds as the standard time-step.

We ran the model for a full year with the time-step range indicated above in order to investigate the impact of the choice of time-step on the realisation of the flow path of the water (and consequently model skill in simulating maximum lake area, onset date and location). We found the model to be generally insensitive to temporal resolution when locating and initiating lakes, with less than $5 \%$ difference between maximum (60 seconds) and minimum (600 seconds) values. All simulations co-located $66 \%$ of observed lakes with an odds ratio of 18-19 and the simulated and observed onset day were found to be strongly correlated with a Pearson correlation co-efficient in the range 0.74-0.76. The most significant impact of a coarser time-step is on the maximum cumulative area; a time-step of 600 seconds was found to have a maximum cumulative area $16 \%$ less that that simulated using a 60 second time-step (Figure A3). A slight sensitivity was observed in the Peirce and Heidke skill scores; found to be 0.33 and 0.32 for the 60 second time-step and 0.30 and 0.29 for the 600 second time-step. This corresponds to a $10 \%$ reduction with a $1000 \%$ increase in timestep and is most likely due to the decrease in maximum cumulative area associated with the increase in time-step. Using a time-step in the 60-120 second range made no significant difference to any of the metrics used to evaluate model skill and so we feel our choice of 90 seconds as the standard time-step is justified. 


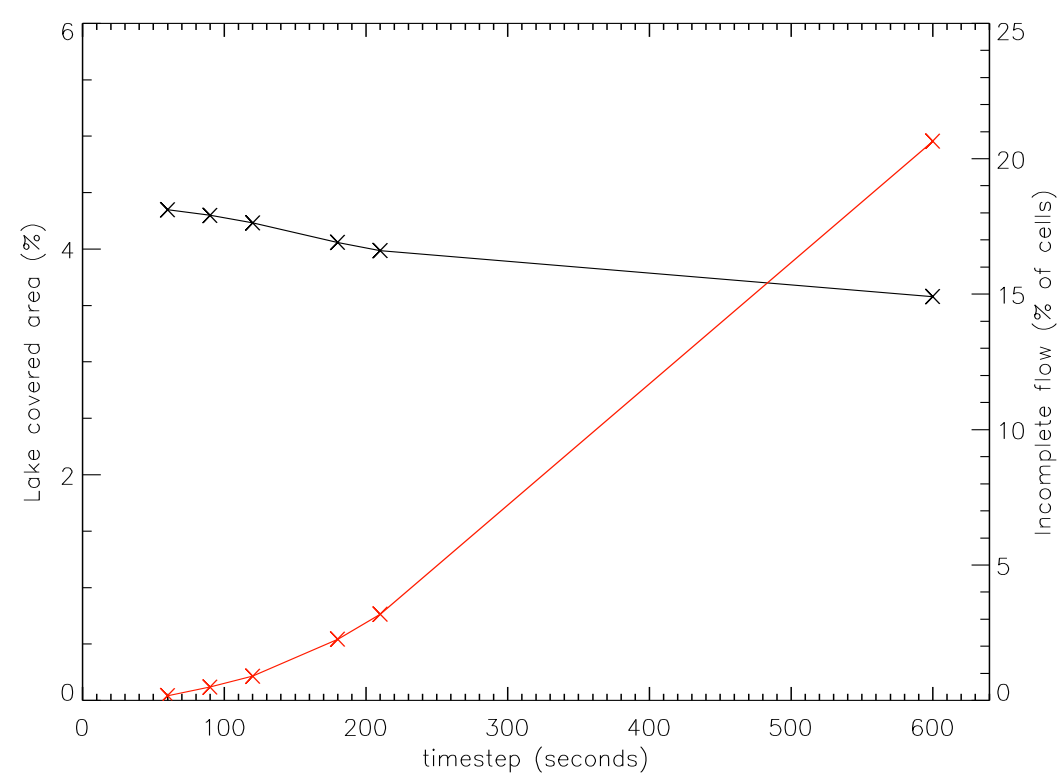

Figure S3: Variation of Lake covered area, as a percentage of the total area, with respect to time-step in seconds (black) and the proportion of cells exhibiting incomplete flow (red)

\section{S4. Sensitivity to temporal resolution of forcing data}

In our model, we apply runoff to the ice sheet at a constant rate using daily values supplied by MAR. In reality, runoff production exhibits a diurnal cycle (Figure A4).

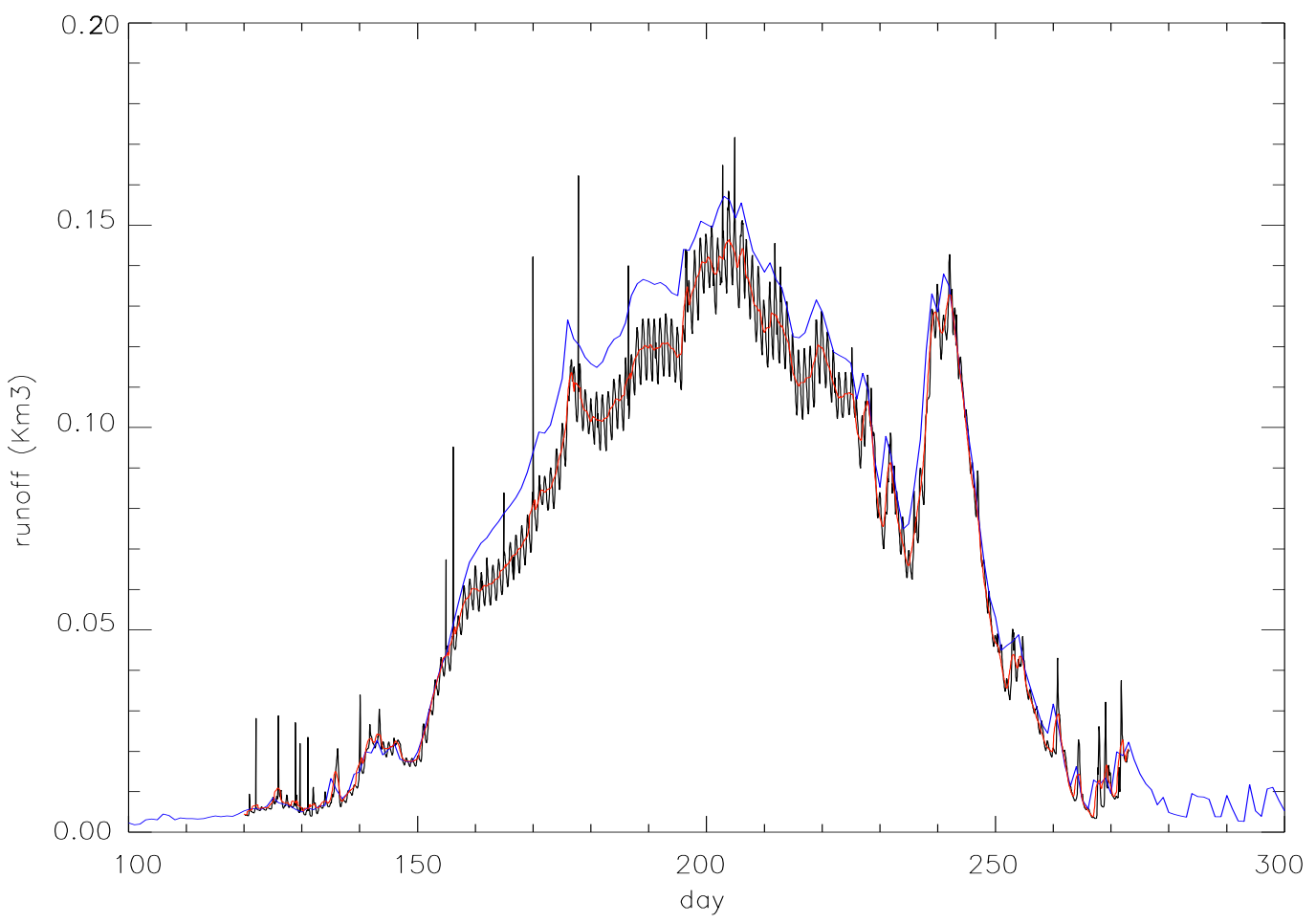

Figure S4 Total hourly runoff for study area from hourly MAR output (black), a low pass filter with a window size of 24 applied to hourly MAR output (red) and daily MAR output (blue) divided by 24. 
The model dependency on this constant rate of runoff was tested by running the model twice using a one hour timestep. The first simulation was forced by hourly MAR runoff rates, and the second simulation was forced by a constant rate of runoff calculated from the summation of hourly MAR output over a 1 day period. We found less that $1 \%$ difference between the simulations in all metrics we apply to assess model skill. This suggests that in our experiments, the accumulation and routing of meltwater over the surface dampens out the diurnal signal of runoff production, with respect to the growth of supra-glacial lakes, and that consequently they exhibit no diurnal variability in their evolution. This is of course, limited to our method of simulating supraglacial lake evolution and may not be the case for lakes which drain.

\section{References}

Chow, V. T., David R. Maidment, Larry W. Mays: Applied Hydrology, McGraw-Hill International Editions; Civil Engineering Series, McGraw-Hill Book Company, Singapore, 1988.

Lotter, G. K.: Considerations on hydraulic design of channels with different roughness of walls, Transactions, All-Union Scientific Research Institute of Hydraulic Engineering, Leningrad, 1932. 